\title{
The publication priorities of Spinal Cord
}

\section{A. Harvey ${ }^{1}$}

Published online: 6 April 2018

(c) International Spinal Cord Society 2018

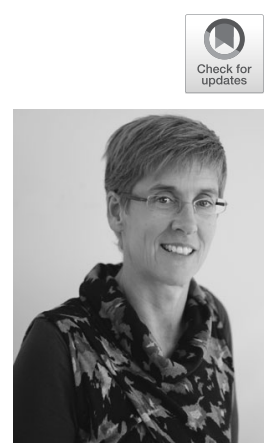

In October 2017, Spinal Cord had its annual face-to-face Editorial Board meeting. At this meeting many things were discussed as we planned the future direction of the journal. However, centre stage was a discussion around our publication priorities. In this Editorial, I want to summarise this discussion and some of the decisions that were made. Interested readers are directed to our updated author guidelines which contain a more detailed explanation of our revised publication priorities.

Spinal Cord is the official scientific journal of the International Spinal Cord Society. So its prime purpose is to publish high quality original research and other papers of scientific value that will be of interest to its members and will progress the care of people with spinal cord injuries. For this reason, we are primarily interested in studies which have clinical implications. This includes studies which investigate the causes and incidence of spinal cord injuries and the secondary consequences of spinal cord injuries, as well as studies which determine the effectiveness of interventions, diagnostic accuracy of tests, prognostic value of clinical measures, psychometric properties of outcomes measures and summaries of evidence. We also value basic science and animal studies, particularly those that have clear relevance to current or future clinical practice.

Spinal Cord has a very broad scope and accepts papers that will be of interest to physicians, scientists, academics, engineers, allied health professionals, nurses, epidemiologists, healthcare managers, economists and many more. The multidisciplinary nature of the journal is one of its unique and valued attributes. It makes the journal a one-stop-read for all aspects of spinal cord injuries.

Spinal Cord prioritises papers that report analyses of qualitative or quantitative data. In other words, Spinal Cord

\footnotetext{
$\triangle$ L. A. Harvey

spinalcord@iscos.org.uk

1 University of Sydney, Sydney, Australia
}

prioritises empirical research. So papers describing different initiatives or clinical programs, or narrative papers outlining historical aspects of the growth of the field is not a publication priority for Spinal Cord. This is not to suggest that such papers are not worthwhile and interesting, just that such papers are not priorities for Spinal Cord.

Our preference is to publish studies which report prospectively collected data. This is not a hard and fast rule Spinal Cord has published and will continue to publish selected retrospective studies that answer important questions. Typically, those are clearly articulated questions about effects of interventions, aetiology (causation) or prognosis. Studies which seek to explore 'relationships' (also expressed as 'associations') are of less interest. Chart audits describing the demographic characteristics of patient cohorts is not a high publication priority unless these data demonstrate an important and previously unrecognised observation or trend.

Spinal Cord is very interested in population-based incidence data and follow-up data on large and representative samples of people with spinal cord injuries. Establishing representativeness requires firstly identifying the population of interest and then either studying all or a random sample from that population. Cohort and observations studies conducted on samples of convenience are problematic and are a low publication priority.

Spinal Cord values hearing the perspectives of people with spinal cord injuries, health professionals and others on various issues. These data are commonly collected through surveys. Therefore, Spinal Cord will consider publishing the results of surveys but it is unlikely to publish surveys of samples of convenience, or surveys with low response rates, regardless of the sample size.

Our publication guidelines arise from a desire to publish reports of rigorous research. We want our readers to be confident that the conclusions and recommendations made in the papers we publish are trustworthy. We rely on studies with good methodology, appropriate statistical analyses and sound interpretation of data. We are particularly keen to see 
the use of methodologies that minimise bias. For example, outcomes assessed by blinded personnel, samples which are representatives of the target population, appropriate statistical analyses, adjustments made for possible sources of confounding, and studies conducted and reported according to pre-planned protocols. These are just some aspects of methodology important for minimising bias: there are of course many others, depending on the type of study.

Lastly, but perhaps most importantly, Spinal Cord prioritises research that answers important questions. We decide if a piece of research is important by asking 'How will the findings of this study change our understanding of spinal cord injury, or the management of spinal cord injury, or the lives of people with spinal cord injuries?'.
It goes without saying that Spinal Cord only publishes papers that adhere to all international standards for the ethical use of animal and human participants. So all papers must clearly state that appropriate ethical approval was attained and that the study followed standards important for the conduct of research on animals and human participants. This applies to all studies, including those in which data are retrospectively collected from medical records.

I hope this editorial helps readers and researchers understand the research priorities of Spinal Cord, and that readers and researchers of Spinal Cord will value the direction the journal is taking in its efforts to publish high quality science and original research that will improve the lives of people with spinal cord injuries. 